\title{
Reduction of hospital stay and cost after the implementation of a clinical pathway for radical gastrectomy for gastric cancer
}

\author{
Jimmy B.Y. So, ZiLiang L. Lim, Heng-An Lin, and Thiow-Kong Ti \\ Department of Surgery, National University Hospital, Yong Loo Lin School of Medicine, National University of Singapore, Lower Kent \\ Ridge Road, 119072 Singapore
}

\begin{abstract}
Background. Clinical pathways have been used for various surgical procedures to improve outcomes and reduce costs. Radical gastrectomy is a major surgery for the treatment of gastric cancer. This study serves to evaluate the difference in clinical outcomes before and after patients were managed on a multidisciplinary gastrectomy pathway.

Methods. Between 2000 and 2005, 115 consecutive patients who underwent gastrectomy were categorized into a prepathway group and a pathway group. This corresponded to the implementation of the clinical pathway in August 2002. The 61 patients in the pathway group were managed according to a multidisciplinary program, while the 54 patients in the pre-pathway group were managed conventionally. The clinical outcomes and hospital costs were then assessed and compared.

Results. The two groups were similar in terms of demographics, comorbidities, types of gastrectomies, and pathological cancer staging. The postoperative length of hospital stay (LOS) was 9 days for the pathway group and 11 days for the pre-pathway group $(P=0.02)$, and the total LOS in the two groups was 11 and 14 days, respectively $(P=0.02)$. The cost of hospitalization for the pathway patients was $\mathrm{S} \$ 13338$, which was significantly lower compared to the $\$ \$ 17371$ cost for the pre-pathway patients $(P=0.047)$.

Conclusion. Clinical pathways may reduce hospital stay and costs for patients undergoing gastrectomy for the treatment of gastric cancer.
\end{abstract}

Key words Gastric cancer · Gastrectomy · Clinical pathway

\section{Introduction}

Gastric cancer remains a leading cause of cancer death worldwide. Gastric resection is the mainstay of curative treatment. However, gastrectomy is a major surgery

Offprint requests to: J.B.Y. So

Received: September 19, 2007 / Accepted: April 12, 2008 with significant perioperative risks. The average postoperative hospital stay is usually $12-18$ days $[1,2]$. Complications are not uncommon, and these further prolong the duration of hospital stay and increase the cost.

Clinical pathways with a multidisciplinary approach have been used to improve the outcomes of patients who undergo major surgery [3, 4]. They aim to provide a coordinated program to accelerate recovery from surgery in a cost-effective manner. Pathways have been used for many procedures, such as colectomies, coronary artery bypasses, and pulmonary resections [5-7]. Since 2002, our unit has implemented a multidisciplinary clinical pathway for all patients who have undergone a gastrectomy. In this study, we evaluated the results of using the pathway and compared the postoperative outcomes before and after its implementation.

\section{Patients and methods}

The study period was from January 2000 to April 2005 and included a total of 115 consecutive patients who underwent elective radical gastrectomy for primary gastric cancer. This cohort consisted of 54 patients in the pre-pathway group and 61 patients in the pathway group. Data were collected retrospectively for the prepathway patients and prospectively for the patients who received the clinical pathway. The data included age, sex, ethnic group, comorbidities, indication for gastrectomy, type of gastrectomy and pathology, length of stay (LOS), and postoperative morbidity and mortality. Readmissions within 1 month after discharge were also recorded. Hospitalization cost data were obtained from the hospital Finance Department.

The gastrectomy pathway was implemented for all gastrectomy patients from August 2002 onwards and was designed to be a multidisciplinary approach to provide cost-effective yet optimal care for these patients, with the main intention of reducing the length of stay 
Table 1. Structure of clinical pathway for gastrectomy

Preoperative
1. Verbal and written preoperative information given by upper gastrointestinal
nurse
2. Counseling and education given by pain nurse, dietitian, and physiotherapist
Perioperative
1. Surgery and postoperative care carried out by upper gastrointestinal surgeons
Postoperative
1. Adequate pain control managed by pain team
2. Aggressive structural mobilization plan
3. Early removal of nasogastric tube
4. Early resumption of diet and protein supplement
5. Discussion of discharge plan with caregiver

and the cost of hospitalization, while maintaining a good clinical outcome (Table 1). To design the clinical pathway, a team of upper gastrointestinal surgeons, a nurse clinician, a dietitian, a physiotherapist, a pain care nurse, and a nurse coordinator was assembled. The team discussed each aspect of patient care and potential intervention. The timing of care was also streamlined in order to make the delivery of care more efficient. There were several components to the clinical pathway; these are described below.

\section{Preoperative education and counseling}

All patients, as well as their family members and caregivers, were given detailed counseling, including written information regarding the gastrectomy, by a trained upper gastrointestinal nurse, to help them understand and prepare themselves for the operation and the perioperative period. An estimated discharge plan was also explained to them. If there were any anticipated difficulties with regards to the discharge of the patient; for example, financial issues and home care, the case may have been referred to a medical social worker.

\section{Pain control}

Preoperatively, all patients were counseled by a trained pain nurse regarding postoperative pain management, including the usage of epidural analgesia and patient controlled analgesia (PCA). Perioperatively, individual anesthetists decided on the type of analgesia to be used. Postoperatively, the hospital pain management team managed the pain control.

\section{Physiotherapy}

Prior to surgery, all patients were educated by a physiotherapist on breathing exercises and the use of incentive spirometries. Cigarette smoking was strongly discouraged. On the first postoperative day, patients were started on a structural mobilization plan to encourage early ambulation.

\section{Nutritional support}

Prior to surgery, all patients were assessed by a dietician. Protein supplements were given if patients were able to tolerate these orally. Postoperatively, liquid and solid diets were restarted early: on day 3 after subtotal gastrectomy and on day 5 after total gastrectomy. Once patients were able to tolerate the diets, they were started on protein supplements.

\section{Surgical care}

All operations were performed and managed by the upper gastrointestinal (UGI) surgical team. Nasogastric tubes were removed on day 1 after total gastrectomy and on day 3 after subtotal gastrectomy. Drains were removed once the output was clear (following the introduction of oral fluids). For the prophylaxis of deep vein thrombosis, all patients received subcutaneous low-molecular weight heparin and wore pressure stockings.

Attending surgeons would initiate the entry of the pathway once the decision for surgery was made; usually at the outpatient clinics and occasionally in the wards. A preprinted checklist including pre- and postoperative orders was used. These checklists covered the routine aspects of patient care such as preoperative tests and postoperative care.

In the pre-pathway group, all patients were managed in the conventional manner by individual surgeons.

\section{Statistics}

All statistical analyses were made using the $\chi^{2}$ test, Student's $t$-test, and an analysis of variance (ANOVA) 
test (where relevant). Results were generated with SPSS (ver 13; Chicago, IL, USA). $P$ values of less than 0.05 $(P<0.05)$ were considered to be significant.

\section{Results}

One hundred and fifteen patients were enrolled in this study; 54 were operated on before the implementation of the clinical pathway for radical gastrectomy for gastric cancer (pre-pathway group) and 61 patients were operated on after the implementation of the pathway in August 2002 (pathway group).

The patients' demographic data and comorbidities are summarized in Table 2 . There were no statistically significant differences between the two groups with regards to age, ethnic group, and comorbidities (except for respiratory comorbidity). A significant proportion of patients in each group had comorbid illnesses.

About one-third of patients in each group received a total gastrectomy. The rest had a subtotal gastrectomy (Table 3). The pathological stages of the cancer were similar in the two groups. Curative or R0 resection was achieved in $88.5 \%$ and $94.4 \%$ of the pathway and the pre-pathway patients, respectively. The number of lymph nodes harvested was greater in the pathway group compared to the pre-pathway group $(P<0.05)$.

The postoperative outcomes in the two groups are summarized in Table 4 . There were three deaths in the whole series $(2.6 \%)$. The reasons for the deaths included bronchopneumonia, acute myocardial infarctions, and anastomotic leakages. There was no mortality in the

Table 2. Demographic data of the patients

\begin{tabular}{lcc}
\hline Demographics & $\begin{array}{c}\text { Pathway group } \\
(n=61)\end{array}$ & $\begin{array}{c}\text { Pre-pathway group } \\
(n=54)\end{array}$ \\
\hline Age, in years (mean) & 66.3 & 63.7 \\
Sex & & 0.265 \\
$\quad$ Male & $43(70.5 \%)$ & $37(68.5 \%)$ \\
Female & $18(29.5 \%)$ & $17(31.5 \%)$ \\
Ethnic group & & 0.818 \\
Chinese & $54(88.5 \%)$ & $49(90.7 \%)$ \\
Malay & $1(1.6 \%)$ & $3(5.6 \%)$ \\
Indian & $1(1.6 \%)$ & $1(1.9 \%)$ \\
Others & $5(8.2 \%)$ & $1(1.9 \%)$ \\
Comorbidities & $31(50.8 \%)$ & $22(45.8 \%)$ \\
Cardiovascular & $5(8.2 \%)$ & $0(0 \%)$ \\
Respiratory & $5(8.2 \%)$ & $1(2.1 \%)$ \\
Renal & $16(26.2 \%)$ & $10(20.8 \%)$ \\
Endocrine & $2(3.3 \%)$ & $0(0 \%)$ \\
Hepatobiliary & $15(24.6 \%)$ & $11(18.5 \%)$ \\
Others & 1.210 & 0.815 \\
Average no. of comorbidities per patient & & 0.321 \\
\hline
\end{tabular}

Table 3. Pathological characteristics

\begin{tabular}{lcc}
\hline Pathological Features & $\begin{array}{c}\text { Pathway group } \\
(n=61)\end{array}$ & $\begin{array}{c}\text { Pre-pathway group } \\
(n=54)\end{array}$ \\
\hline Stage (AJCC) & & \\
0 & $0(0 \%)$ & $1(2 \%)$ \\
1 & $17(28 \%)$ & $11(20 \%)$ \\
2 & $19(31 \%)$ & $13(24 \%)$ \\
3 & $15(25 \%)$ & $19(35 \%)$ \\
4 & $10(16 \%)$ & $10(19 \%)$ \\
R0 resection & & $51(94.4 \%)$ \\
Yes & $54(88.5 \%)$ & $3(5.6 \%)$ \\
No & $7(11.5 \%)$ & 18.7 \\
No. of lymph nodes harvested (mean) & 23.4 & $14(25.9 \%)$ \\
Type of operation & & $40(74.1 \%)$ \\
Total gastrectomy & $14(23 \%)$ & $0(0 \%)$ \\
Subtotal gastrectomy & $45(74 \%)$ & 0.483 \\
Others & $2(3 \%)$ & $<0.05$ \\
\hline
\end{tabular}


Table 4. Postoperative outcomes

\begin{tabular}{lccr}
\hline Postoperative outcome & $\begin{array}{c}\text { Pathway group } \\
(n=61)\end{array}$ & $\begin{array}{c}\text { Pre-pathway group } \\
(n=54)\end{array}$ & $P$ value \\
\hline Mortality during hospitalization & $0(0 \%)$ & $3(5.6 \%)$ & 0.062 \\
Complication rate & $24(39.3 \%)$ & $21(38.9 \%)$ & 0.960 \\
Mean total LOS (days) & 11.29 & 14.04 & 0.023 \\
Mean postop LOS (days) & 8.88 & 11.00 & 0.022 \\
Re-admission rate (30-day) & $11(18 \%)$ & $7(13.0 \%)$ & 0.455 \\
Cost (mean; S\$) & 13337.90 & 17371.44 & 0.047 \\
\hline
\end{tabular}

Table 5. Reasons for readmission

\begin{tabular}{lcc}
\hline & $\begin{array}{c}\text { Pathway group } \\
(n=11)\end{array}$ & $\begin{array}{c}\text { Pre-pathway group } \\
(n=7)\end{array}$ \\
\hline Inadequate oral feeding & 3 & 2 \\
Constipation & 1 & 1 \\
Fever & 1 & 0 \\
Pneumonia & 3 & 1 \\
Anastomotic failure & 1 & 0 \\
Intraabdominal abscess & 1 & 0 \\
Angina pectoris & 0 & 1 \\
Anal fissure & 0 & 1 \\
Creation of AVF & 1 & 0 \\
Palliative care & 0 & 1 \\
\hline
\end{tabular}

pathway patients. Total and postoperative length of stay (LOS) in the hospital were both significantly shorter in the pathway patients. The mean postoperative LOS for the pathway patients was 9 days, as compared to 11 days in the pre-pathway group $(P=0.02)$. The difference in mean postoperative LOS was 2.12 days (95\% confidence interval $[\mathrm{CI}], 0.32$ to 3.92 ). The difference in mean total LOS was 2.75 days (95\% CI, 0.39 to 5.11). The mean total hospitalization cost for the pathway patients was significantly lower, at $\mathbf{S} \$ 13338$, as compared to $\mathrm{S} \$ 17371$ in the pre-pathway patients $(P<0.05)$, with the mean difference in cost being $\mathrm{S} \$ 4033$ (95\% CI, 58 to 8,009$)$.

Within 30 days after discharge, there were 11 (18.0\%) readmissions in the pathway group and $7(13.0 \%)$ in the pre-pathway group; the difference was not statistically significant $(P=0.455)$. The reasons for readmission are shown in Table 5.

\section{Discussion}

Our study demonstrated that a structured clinical pathway reduced both the hospital stay and the cost for patients undergoing gastrectomy for cancer. It is the first study in the literature to evaluate a multidisciplinary perioperative program for gastrectomy patients. We have also shown that radical gastrectomy is a safe procedure with acceptable mortality and morbidity risks, when performed in a specialized unit.
As the cost of healthcare escalates rapidly over the years, means to reduce the costs of treatment are highly sought after. Clinical pathways have been developed to standardize the care for patients with a particular medical condition, or after a procedure. Such pathways minimize the variations in management by different care providers. To construct a clinical pathway, inputs from various disciplines are collected and decisions are made based on evidence-based medicine. In the past decade, pathways with a multimodal optimization program have been implemented for many types of major surgery, such as colectomies, cardiac bypasses, and pulmonary resections, with favorable results. The key principles of these enhanced recovery programs are adequate analgesia, early ambulation, and early resumption of oral intake [5, 8].

Radical gastrectomy is a major surgical procedure, with significant risks of morbidity and mortality $[1,2,9-$ 11]. Advanced age, coexisting illnesses, and malnutrition are common among gastric cancer patients. These factors further increase the risk of surgery [4]. In addition, upper abdominal incision is associated with significant postoperative pain, resulting in subsequent pulmonary atelectasis [12]. Nutritional counseling and support is one of our key components in this pathway. Dietitians, clinicians, and nurse clinicians are all involved. Prior to surgery, patients receive a high-protein diet with supplements whenever possible, as early satiety and malnutrition are common after gastrectomy [13]. Upon discharge from surgery, detailed counseling and visual 
information on diet are given to patients and their families. Chest physiotherapy with incentive spirometry is practiced aggressively before and after the surgery to prevent pulmonary atelectasis. Pain management carried out by a specialized team plays an integral part in our pathway in minimizing postoperative pain and its associated complications. The nurse clinician then coordinates various components of the pathway.

There are some limitations in this study. Patients in the study were not randomized. However, we compared all patients immediately before and after implementation of the pathway for the study. The two groups were also comparable in terms of demographics and pathological features. It is difficult to conduct a randomized study nowadays as the institution of a clinical pathway would also influence the care of patients not on the pathway. Another introduced bias is the standard of surgery. Prior to the pathway period, both general and upper gastrointestinal surgeons performed gastrectomies. With the introduction of the pathway, patients in this group had gastrectomies performed only by two sub-specialist upper gastrointestinal surgeons [14]. This change of practice in our unit may have affected the operative standards, as suggested by the increased extent of lymph node dissection (mostly D2) in the pathway group [15]. However, extended nodal dissection may increase the risk of postoperative complications, which would favor the results of the pre-pathway group. In spite of this, patients in the pathway group had a shorter hospital stay with lower cost than those in the pre-pathway group.

One of the potential drawbacks in the pathway group was the readmission after discharge from the hospital [3]. Most readmissions were due to minor problems, some of which could have been avoided. Currently, all patients are assessed 1 day post-discharge via a telephone interview by our nurse clinician. They are also reviewed at the outpatient clinics within a week. These measures help to identify any postoperative problems early and, thus, avoid unnecessary readmissions to the hospital.

In summary, a clinical pathway with a multidisciplinary program improves the outcome of patients undergoing gastrectomy for gastric cancer. This pathway reduces both the length of hospitalization and the costs involved for that particular surgery.

\section{References}

1. Park DJ, Lee HJ, Kim HH, Yang HK, Lee KU, Choe KJ. Predictors of operative morbidity and mortality in gastric cancer surgery. Br J Surg 2005;92:1099-102.

2. Cuschieri A, Fayers P, Fielding J, Craven J, Bancewicz J, Joypaul $V$, et al. Postoperative morbidity and mortality after $D_{1}$ and $D_{2}$ resections for gastric cancer: preliminary results of the MRC randomized controlled surgical trial. Lancet 1996;347:995-9.

3. Kehlet H, Wilmore DW. Fast track surgery. Br J Surg 2005;92: 3-4.

4. Kehlet H, Wilmore DW. Multimodal strategies to improve surgical outcome. Am J Surg 2002;183:630-41.

5. Gatt M, Anderson AD, Reddy BS, Hayward-Sampson P, Tring IC, MacFie, J. Randomized clinical trial of multimodal optimization of surgical care in patients undergoing major colonic resection. Br J Surg 2005;92:1354-62.

6. Bohmer RM, Newell J, Torchiana DF. The effect of decreasing length of stay on discharge destination and readmission after coronary bypass operation. Surgery 2002;132:10-5.

7. Tovar EA, Roethe RA, Weissig MD, Lloyd RE, Patel GR. Oneday admission for lung lobectomy: an incidental result of a clinical pathway. Am Thorac Surg 1998;65:803-6.

8. Kehlet H, Dahl JB. Anaesthesia, surgery, and challenges in postoperative recovery. Lancet 2003;362:1921-8.

9. Kodera Y, Sasako M, Yamamoto S, Sano T, Nashimoto A, Kurita A. Identification of risk factors for the development of complications following extended and superextended lymphadenectomies for gastric cancer. Br J Surg 2005;92:1103-9.

10. Sasako M. Risk factors for surgical treatment in the Dutch Gastric Cancer Trial. Br J Surg 1997;84:1567-71.

11. Wu CW, Hsiung CA, Lo SS, Hsieh MC, Shia LT, Whang-peng J. Randomized clinical trial of morbidity after D1 and D3 surgery for gastric cancer. Br J Surg 2004;91:283-7.

12. Inaba T, Okinaga K, Fukushima R, Iinuma H, Ogihara T, Ogawa $\mathrm{F}$, et al. Prospective randomized study of two laparotomy incisions for gastrectomy: midline incision versus transverse incision. Gastric Cancer 2004;7:167-71.

13. Hirao M, Tsujinaka T, Takeno A, Fujitani K, Kurata M. Patientcontrolled dietary schedule improves clinical outcome after gastretomy for gastric cancer. World J Surg 2005;29:853-7.

14. Ti TK. Radical surgery for gastric cancer in Singapore. Surg Today Jpn J Surg 1995;25:573-8.

15. Callahan MA, Christos PJ, Gold HT, Mushlin AI, Daly JM. Influence of surgical subspecialty training on in-hospital mortality for gastrectomy and colectomy patients. Ann Surg 2003;238: 629-39. 